\title{
Acute Acalculous Cholecystitis in Neurological Patients; Clinical Review, Risk Factors, and Possible Mechanism
}

\author{
See Won Um, Hak Cheol Ko, Seung Hwan Lee, Hee Sup Shin, Jun Seok Koh \\ Department of Neurosurgery, Kyung Hee University Hospital at Gangdong, College of Medicine, Kyung Hee University, Seoul, Korea
}

Received: August 26, 2019

Accepted: September 10, 2019

Corresponding Author:

Hak Cheol Ko, M.D., Ph.D.

Department of Neurosurgery,

Kyung Hee University Hospital at

Gangdong, 892, Dongnam-ro,

Gangdong-gu, Seoul 05278,

Korea

Tel: +82-2-440-8402

Fax: +82-2-440-8404

E-mail: neurosko@gmail.com

\section{Backgrounds}

Acute acalculous cholecystitis (AAC) has rarely been reported and has not been fully investigated in patients with severe neurological conditions that can cause changes in consciousness. Delays in diagnosis of AAC may increase the severity of this condition. The aim of this study was to investigate the clinical features and possible mechanisms of AAC in neurological patients.

\section{Methods}

Among the patients with neurological conditions admitted to our hospital between March 2007 and September 2018, the medical records of 52 patients who underwent cholecystectomy after being diagnosed with cholecystitis were reviewed retrospectively. Data regarding age, sex, neurological conditions, Glasgow Coma Scale (GCS) on admission, past medical history, time from admission to the onset of AAC, initial symptoms or signs of AAC, and fasting period were investigated.

\section{Results}

The mean age of the 52 patients ( 36 male, 16 female) was $67.1 \pm 14.4$. The mean GCS was 10 . The neurological conditions were intracerebral hemorrhage, subarachnoid hemorrhage, subdural hematoma, intraventricular hemorrhage, epidural hematoma, cerebral infarction, cerebral abscess, and hypoxic brain damage. The mean time interval between the onset of AAC and admission was 22.5 days and the mean fasting period was 8.1 days.

\section{Conclusion}

AAC may be a significant complication in patients with neurological conditions. It is important to identify symptoms and signs of AAC, accurately diagnose the AAC, particularly in long-term stay at intensive care unit (ICU), a long period of fasting, state of hypotension or hypoperfusion, high positive end-expiratory pressure (PEEP) ventilation.

Keywords: Cholecystitis; Neurological Intensive Care Unit; Mechanism

\section{INTRODUCTION}

Acute cholecystitis is known to be caused by the pathologic mechanism of inflammation and ischemia in the gallbladder (GB) wall $^{1)}$, stagnant pooling of bile juice, and the lithogenicity of bile juice in a complex manner. The incidence of acute cholecystitis is

Copyright (C) 2019 The Korean Neurointensive Care Society

This is an Open Access article distributed under the terms of the Creative Commons Attribution Non-Commercial License (http://creativecommons.org/licenses/by-nc/4.0/) which permits unrestricted non-commercial use, distribution, and reproduction in any medium, provided the original work is properly cited. 
especially high after a major surgery or serious illness. Acute cholecystitis may occur in the presence of a GB stone, however acute acalculous cholecystitis (AAC) is reported to occur more often ${ }^{2}$.

Hypoperfusion and bile stasis are the key pathogenesis of AAC, which could be aggravated by hypotension, dehydration, and the usage of vasoactive drugs. Bile stasis is caused by the use of opioids, fasting with total parenteral nutrition (TPN), and mechanical ventilation with positive end-expiratory pressure (PEEP) ${ }^{3)}$.

$\mathrm{AAC}$ is reportedly a complication of a disease with high mortality unless treatment, long-term and short-term mortality may reach $59 \%$ and $35 \%$, respectively ${ }^{4)}$. The incidence of AAC is rare in patients with severe neurological conditions with alterations in consciousness, such as cerebrovascular disease (CVD) or severe brain trauma. However, diagnosing and treating $\mathrm{AAC}$ are important in avoiding high mortality. Therefore, it is important to recognize the symptoms and signs of AAC, make an accurate diagnosis, and provide expedient treatment. Neurological patients are accompanied by severe neurological symptoms and signs, such as loss of consciousness, paraplegia, and aphasia, which can increase the severity of the symptoms of AAC.

Nevertheless, abdominal evaluations are not routinely done in patients with CVD or brain trauma and there are no established clinical characteristics of AAC in the intensive care unit (ICU). The aim of this study is to investigate the clinical features of AAC and the possible mechanisms of AAC in neurological patients.

\section{METHODS}

Among the patients with neurological conditions admitted to our hospital between March 2007 and September 2018, the medical records of 52 patients who underwent cholecystectomy after being diagnosed with cholecystitis were reviewed retrospectively. Data regarding age, sex, neurological conditions, mental status on admission, past medical history, time from admission to the onset of AAC, initial symptoms or signs of AAC, and fasting period were investigated.

AAC was diagnosed through abdominal sonography or computed tomography (CT), which showed no gallstones. The date of diagnosis was defined as the day in which AAC was diagnosed through the abdominal imaging.

\section{RESULTS}

The clinical characteristics of total 52 patients who underwent cholecystectomy is summarized in Table 1 . The average age was 67.1 years with broad age spectrum spanning from 17 to 90 yearsold, only one 17 years-old, no one in the 20 s, 17 in the 60 s, and 19 in the 70s with the age group in their 70s showing the highest prevalence. There were 36 males and 16 females with the males showing 1.89 times higher incidence than the females. For the underlying disease, there were HTN in 23 patients, diabetics mellitus (DM) in 10 patients. The mean Glasgow Coma Scale (GCS) was 10 which implies neurological deterioration with drowsy to stuporous mentality being the most prevalent. Also, Intracerebral hemorrhage was the most common neurological diagnosis among brain insults with subarachnoid hemorrhage, subdural hemorrhage, and epidural hemorrhage in order of descent.

The clinical factors related to AAC are summarized in Table 2. The mean time from admission to onset of AAC was 22.5 days and length of hospital stay showed an average of 77.9 days. The mean fasting period was 8.1 days. The initial symptoms and signs of AAC were elevation of liver function test (LFT), which was also the most common symptoms and signs in 27 patients; there were right upper quadrant (RUQ) pain or tenderness in 4 patients, fever, in 2 patients, C-reactive protein (CRP) elevation in 5 patients, and incidentally diagnosed in 10 patients. The 10 incidentally diagnosis of AAC was done through abdominal sonography or CT after suspicions of acute kidney injury, cystitis, pneumonia, and pleural effusion. The asymptomatic 10 patients received cholecystectomy

Table 1. Clinical characteristics of 52 patients

\begin{tabular}{lc}
\hline Characteristics & \\
Sex (number) & 36 \\
Male & 16 \\
Female & 67.1 \\
Age (mean) & \\
Past medical history & 23 \\
Hypertension & 10 \\
Diabetes mellitus & 2 \\
Cerebrovascular disease & 5 \\
Heart disease & 4 \\
Alcoholics & \\
Glasgow Coma Scale on admission & 26 \\
$\geq 13$ & 4 \\
7 -12 & 22 \\
<6 & \\
Neurological conditions & 12 \\
Subarachnoid hemorrhage & 19 \\
Intracerebral hemorrhage & 11 \\
Subdural hematoma & 3 \\
Epidural hematoma & 8 \\
Intraventricular hemorrhage & 3 \\
Cerebral infarction & 1 \\
Hypoxic brain damage & 2 \\
Cerebral abscess & \\
& \\
\hline &
\end{tabular}


after discussion among board certified gastroenterologists or general surgeons. Of the patients who were treated for neurological diseases, there were 35 patients who have received neurosurgical operative treatment and 17 patients who have received conservative management. Of which the group received neurosurgical operative treatment had a higher prevalence of AAC.

The patients who have received cholecystectomy after being diagnosed with AAC were divided into the neurosurgical treatment group and the conservative management group (Table 3). The mean time from admission to onset of AAC in the neurosurgical treatment group was 27.3 days, which was 14.7 days longer than the conservative management group (12.6 days). The mean fasting period in the neurosurgical treatment group was 9.1 days and the that in the conservative management group was 6.1 days. Mean

Table 2. Clinical factors associated with acute acalculous cholecystitis

\begin{tabular}{lc}
\hline Clinical factors & \\
\hline Mean time from admission to onset of AAC (day) & 22.5 \\
Hospital stay (day) & 77.9 \\
Mean fasting period (day) & 8.1 \\
Initial symptoms or signs (number) & \\
$\quad$ RUQpain or tenderness & 8 \\
Fever & 2 \\
Elevation of LFT & 27 \\
Elevation of CRP & 5 \\
$\quad$ Diagnosis incidentally & 10 \\
Neurological treatment & \\
Operative & 35 \\
Conservative & 17 \\
\hline
\end{tabular}

AAC: acute acalculous cholecystitis; CRP: c-reactive protein; LFT: liver function test; RUQ: right upper quadrant.
GCS in the neurosurgical treatment and conservative management group were 9.4 and 12.1, respectively.

\section{DISCUSSION}

The prevalence of cholecystitis in GB stone patients after surgery is reported to be similar between male and female. However, over $80 \%$ of AAC after other operations unrelated to trauma are male patients aged 50 years old and over ${ }^{5}$. This indicates that emergent operation or an elective operation complicated by hypotension and blood loss is pronounced in male patients ${ }^{2}$. The incidence of AAC in this study showed a 1.89 times higher rate of incidence in males than in females as noted. This could be explained that the rate of comorbidities is higher in the male patients ${ }^{6}$, however sex alone cannot be considered an independent factor for cholecystectomy in neurological patients. Nevertheless, special attention could be paid to the occurrence of AAC in male patients considering what is reported in previous studies and this study.

Most of the neurological deteriorated patients admitted to the ICU requires PEEP, which can be associated with bile stasis ${ }^{7)}$. $7-10 \mathrm{~cm}$ ventilation in $\mathrm{H}_{2} \mathrm{O}$ PEEP can raise hepatic venous pressure, which causes a decrease in portal perfusion ${ }^{7}$, in turn can cause bile stasis and increases the possibility of gallbladder mucosal injury. There was no statistically significant difference among the patients in this study as $4-5 \mathrm{~cm}$ ventilation of $\mathrm{H}_{2} \mathrm{O}$ PEEP was applied, but it is recommended to always keep in mind the possibility of bile stasis in patients requiring $7-10 \mathrm{~cm}$ high PEEP.

The average duration in ICU stay was 13.3 days with mean fasting period 7.3 days. Yang et al. ${ }^{8)}$ reported that successive fasting time as a risk factor of acute cholecystitis in aneurysmal subarachnoid hemorrhage patients. Successive fasting time reported was

Table 3. Clinical factors associated with acute acalculous cholecystitis

\begin{tabular}{lcc}
\hline Variables & Neurosurgical treatment $(\mathrm{n}=35)$ & Conservative management $(\mathrm{n}=17)$ \\
\hline Mean time from admission to onset of AAC (day) & 27.3 & 12.6 \\
Mean fasting period (day) & 9.1 & 6.1 \\
Mean GCS on admission & 9.4 & 12.1 \\
Neurological conditions & & 2 \\
$\quad$ Subarachnoid hemorrhage & 10 & 8 \\
Intracerebral hemorrhage & 11 & 2 \\
Subdural hematoma & 9 & 2 \\
Epidural hematoma & 1 & 1 \\
Intraventricular hemorrhage & 7 & 2 \\
Cerebral infarction & 1 & 1 \\
Hypoxic brain damage & - & 2 \\
Cerebral abscess & 2 & -
\end{tabular}

AAC: acute acalculous cholecystitis; GCS: Glasgow coma scale. 
$5.38 \pm 2.78$ days and the pathogenesis of acute cholecystitis included bile stasis as a result of fasting time as its mechanism. Loss of enteric stimulation during parenteral nutrition impairs the gallbladder motor function and causes GB "sludge" formation. Long-term TPN can increase the incidence of AAC by up to $30 \%{ }^{7)}$, GB "sludge" formation can occur in about $50 \%$ of patients treated with TPN for 4 weeks and in $100 \%$ of patients after 6 weeks ${ }^{9}$. Autonomic nerve dysfunction in neurological patients may cause bile stasis. Sevastos et al. ${ }^{10)}$ was proved to cause abnormalities in the flow control of bile and pancreatic juice through increase in vagal paresis and catecholamine secretion following the damage of the brain center with the cessation of oral feeding.

Gallbladder ischemia is pathogenesis of AAC. Orlando et al. ${ }^{11)}$ reported that gallbladder ischemia and bile stasis leading to hypoperfusion. Intraluminal pressure is increased by bile stasis, which results in a decrease in gallbladder perfusion pressure. In this hypothesis, bacterial invasion of ischemic tissue is a secondary phenomenon, acute bacterial and viral diseases, such as hepatitis, infection of upper respiratory tract, are important precipitating factors $^{11-14)}$. Gallbladder ischemia increases mucosal phospholipase A2 and superoxide dismutase activities, and mucosal lipid peroxide content. Whereas gallstone-related disease is associated with arterial dilation and extensive venous filling, but AAC is associated with arterial occlusion and minimal to absent venous filling ${ }^{15)}$.

Another mechanism closely related to the pathogenesis of AAC is systemic inflammatory response. The response to systemic injury and subsequent release of inflammatory mediator, such as factor XII, platelet-activating factor, induce the inflammatory response of the gallbladder ${ }^{5}$. The neurological patients admitted to the ICU is prone to lung problems, including pneumonia, atelectasis, pleural effusion, catheter-related infections, operations-related infection, drug fever, inflammation due to hemorrhage without infectious causes, pseudomembranous colitis, bacteremia, and urinary tract infection (UTI), which could be related to the systemic inflammatory response. Pneumonia and UTI took up more than half of the infections in the ICU. Systemic inflammatory response can cause cholestasis, gallbladder ischemia, therefore could affect the incidence of AAC in neurological patients ${ }^{2)}$.

\section{CONCLUSION}

AAC may be a significant complication in patients with neurological conditions. It is important to identify symptoms and signs of AAC, accurately diagnose the AAC, particularly in long-term stay at ICU, a long period of fasting, state of hypotension or hypoperfusion, PEEP ventilation.

\section{NOTES}

\section{Conflict of interest}

No potential conflict of interest relevant to this article was reported.

\section{REFERENCES}

1. Naidech AM, Bendok BR, Bernstein RA, Alberts MJ, Batjer $\mathrm{HH}$, Watts CM, et al. Fever burden and functional recovery after subarachnoid hemorrhage. Neurosurgery 2008;63:212217; discussion 217-8.

2. Barie PS, Eachempati SR. Acute acalculous cholecystitis. Curr Gastroenterol Rep 2003;5:302-309.

3. Kim SJ, Lee SJ, Lee SH, Lee JH, Chang JH, Ryu YJ. Clinical characteristics of patients with newly developed acute cholecystitis after admission to the intensive care unit. Aust Crit Care 2019;32:223-228.

4. Glenn F, Becker CG. Acute acalculous cholecystitis. An increasing entity. Ann Surg 1982;195:131-136.

5. Delemos AS, Friedman LS. Systemic causes of cholestasis. Clin Liver Dis 2013;17:301-317.

6. Nikfarjam M, Harnaen E, Tufail F, Muralidharan V, Fink MA, Starkey G, et al. Sex differences and outcomes of management of acute cholecystitis. Surg Laparosc Endosc Percutan Tech 2013;23:61-65.

7. Kouroumalis E, Hopwood D, Ross PE, Milne G, Bouchier IA. Gallbladder epithelial acid hydrolases in human cholecystitis. J Pathol 1983;139:179-191.

8. Yang NR, Hong KS, Seo EK. Acute cholecystitis as a cause of fever in aneurysmal subarachnoid hemorrhage. Korean J Crit Care Med 2017;32:190-196.

9. Messing B, Bories C, Kunstlinger F, Bernier JJ. Does total parenteral nutrition induce gallbladder sludge formation and lithiasis? Gastroenterol 1983;84:1012-1019.

10. Sevastos N, Savvas SP, Rafailidis PI, Manesis EK. Cholestasis in acute stroke: an investigation on its prevalence and etiology. Scand J Gastroenterol 2005;40:862-866.

11. Orlando R, Gleason E, Drezner AD. Acute acalculous cholecystitis in the critically ill patient. Am J Surg 1983;145:472-476.

12. Souza LJ, Braga LC, Rocha Nde S, Tavares RR. Acute acalculous cholecystitis in a teenager with hepatitis a virus infection: a case report. Braz J Infect Dis 2009;13:74-76.

13. Imamoglu M, Sarihan H, Sari A, Ahmetoglu A. Acute acalculous cholecystitis in children: Diagnosis and treatment.J Pediatr Surg 2002;37:36-39.

14. Parithivel VS, Gerst PH, Banerjee S, Parikh V, Albu E. Acute 
acalculous cholecystitis in young patients without predisposing factors. Am Surg 1999;65:366-368.

15. de Oliveira Júnior SA, Lemos TEV, de Medeiros Junior AC, Freire AD, de Carvalho Garcia C, e Silva R, et al. Acute Acalcu- lous Cholecystitis in critically ill patients: risk factors, diagnosis and treatment strategies. J Pancreas 2016;17:580-586. 